\title{
First-order imprinted organic distributed feedback lasers
}

\author{
Dario Pisignano $^{\mathrm{a}, *}$, Luana Persano ${ }^{\mathrm{a}, \mathrm{b}}$, Elisa Mele ${ }^{\mathrm{a}, \mathrm{b}}$, Paolo Visconti ${ }^{\mathrm{a}}$, Marco Anni ${ }^{\mathrm{a}}$, Giuseppe \\ Gigli $^{a}$, Roberto Cingolani ${ }^{a}$, Laura Favaretto ${ }^{c}$, Giovanna Barbarella ${ }^{c}$ \\ ${ }^{a}$ NNL, National Nanotechnology Laboratory of Istituto Nazionale di Fisica della Materia (INFM), c/o Dipartimento di Ingegneria \\ dell'Innovazione, Università di Lecce, via Arnesano, I-73100 Lecce, Italy \\ ${ }^{\mathrm{b}}$ ISUFI, Innovative Materials and Technologies School, Università di Lecce, via Arnesano, I-73100 Lecce, Italy

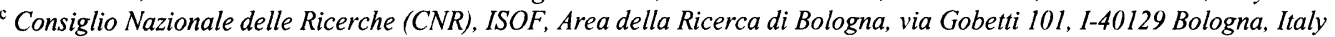

\begin{abstract}
The excellent luminescence and gain properties of substituted oligothiophenes are exploited to imprint one-dimensional photonic crystals for the fabrication of printed distributed feedback (DFB) cavities. We demonstrate the $d r y$-printing of organic semiconductor lasers, carrying out the realization of a complete device by a single nanofabrication step, which can be perfectly integrated with other existing planar semiconductor processing and etching technologies. In particular, a thiophene-based pentamer with very good amplified spontaneous emission and optical gain properties was used to fabricate a single-mode, first-order DFB lasers exhibiting an emission with a linewidth of $1.1 \mathrm{~nm}$ at about $636 \mathrm{~nm}$ for excitation fluences larger than $27 \mu \mathrm{J} / \mathrm{cm}^{2}$. These results clearly indicate that substituted oligothiophene-S,Sdioxides are promising candidates for solid-state lasers realizable by emerging nanopatterning technologies.
\end{abstract}

Keywords: Lasers; Stimulated luminescence; Spin wasting; Manipulation of surface structure and morphology.

\section{Introduction}

Light-emitting organic materials have attracted an increasing interest for the realization of active optical devices for the high photoluminescence (PL) efficiency, wide tunability of the emission, low cost and simple deposition and processing techniques. In particular, the optical gain observed in many different organic compounds, both in solution [1] and in thin-films [2], can be employed for the fabrication of organic semiconductor lasers. A number of conjugated compounds have been demonstrated to show optical gain [3-7], and optically pumped lasing has been achieved with different cavity geometries [8-13]. Among these compounds, functionalized thiophene-based oligomers are interesting, because of their wide colour tunability $[14,15]$, good chemical stability [16], high solubility in organic solvents and good filmforming capability.

Unfortunately, the low molar mass of these compounds strongly limits their use with high-temperature embossing techniques, due to their poor thermoplastic behaviour. In particular, the nanoimprint lithography (NIL)

\footnotetext{
* Corresponding author. Tel: +39-0832-298146; fax: +39-0832-

298238; E-mail: dario.pisignano@unile.it
}

[17] exploits the glass transition of polymers to transfer with very high fidelity master patterns realized by conventional lithographic techniques. It employs a rigid mold to directly pattern micro- and nanostructures into target polymers, with no need of exposure to radiation, development and etching processes, offering the large-area and low-cost operation typical of mechanical lithographies [18]. Direct three-dimensional patterning [19], nanoscale field effect transistors [20], metal-semiconductor-metal photodetectors [21], and sub-10 nm structures have been so far demonstrated [22].

During the imprinting, the mold is placed onto a cast film of the target polymer. The overall system is then driven at a temperature considerably higher than the glass transition temperature, $T_{g}$, of the target. Once at the moldable state, the target material takes the shape of the pattern in the master. The subsequent cooling of the system below $T_{g}$ freezes the pattern on the target surface, thus providing a negative copy of the master. Such a thermal cycle is an intrinsic problem of conventional NIL, as it requires a thermoplastic behaviour of the target compound which can be hardly achieved in small molecules, thus preventing their use as directly printed active materials.

Moreover, since heating the samples can cause the degradation of the optical and electrical properties of the 


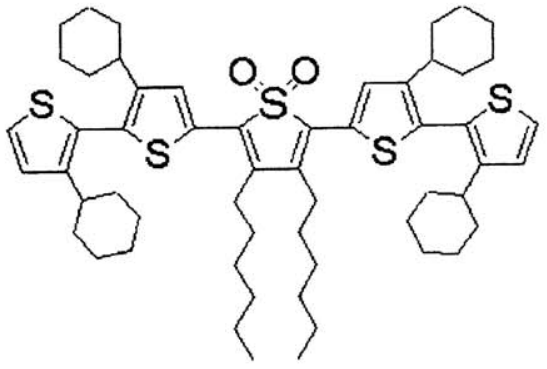

Fig. 1 Molecular structure of the employed oligothiophene- $S, S$ dioxide.

organic materials NIL has to be performed under vacuum to pattern light-emitting molecules [23], and roomtemperature processes have been proposed for conductive polymers, including imprinting [23] and micromolding in capillaries [24].

\section{Experimental}

Films of the substituted oligothiophene, 3,3',4,',3,','Tetracyclehexyl-3', 4 "'

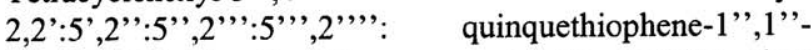
dioxide (T5oCx, Fig. 1) were cast onto Corning glass substrates, from chloroform solutions. Master patterns with

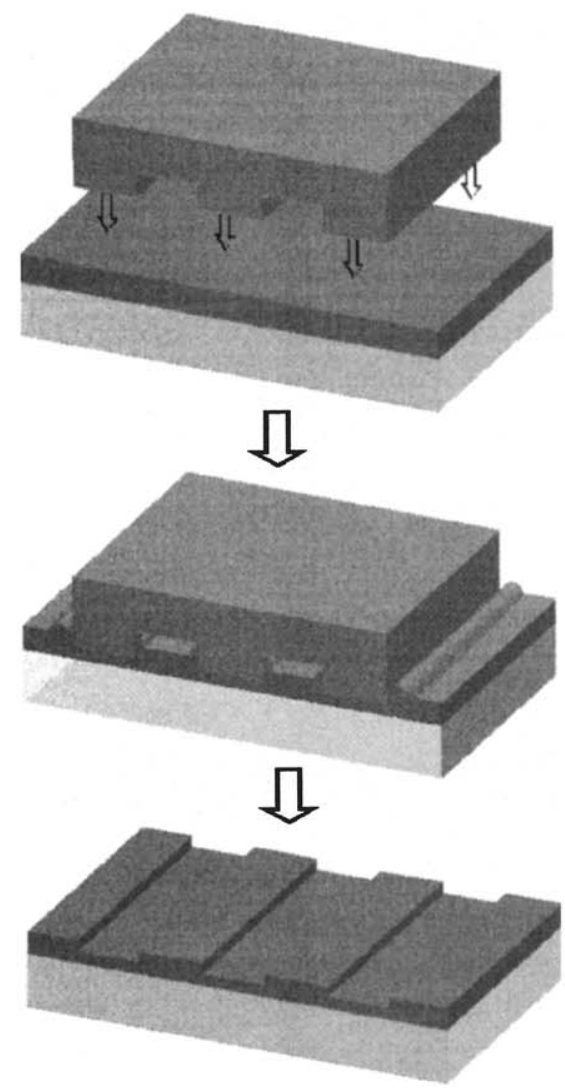

Fig. 2 Scheme of the room-temperature nanoimprinting.

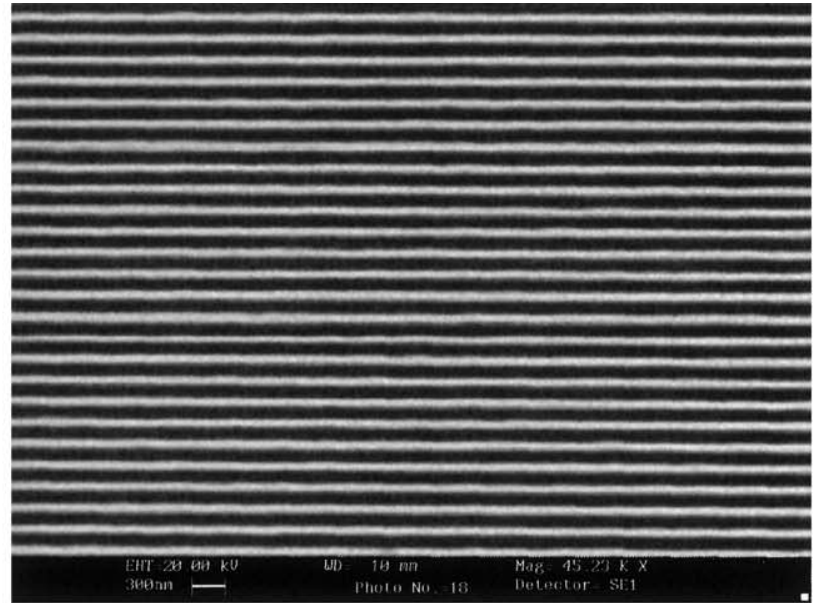

Fig. 3 Si master grating imaged by Scanning Electron Microscopy.

a period, $\Lambda=200 \mathrm{~nm}$, and an area of $1 \mathrm{~mm} \times 6 \mathrm{~mm}$ were first fabricated on Silicon by electron beam lithography with a Leica LION LVI system working at acceleration energy of $2.5 \mathrm{keV}$, and subsequent lift-off and conventional reactive ion etching by a $\mathrm{CF}_{4} / \mathrm{Ar}$ mixture chosen in order to minimize the plasma-induced polymer deposition. The subsequent procedure of nanoimprint lithography at room temperature is schematised in Fig. 2. The master was directly used as a stamp for nanoimprint, employing a precision manual press (PW100 P/O/Weber, Germany) at room temperature, in air (Fig. 2), with an applied force of a few $\mathrm{kN}$. No antisticking layer was needed in the imprinting process. After separating the stamp from the patterned sample, the obtained DFB device was excited by the third harmonic $(\lambda=355 \mathrm{~nm})$ of a $3 \mathrm{~ns} Q$-switched Nd:YAG laser (Spectra-Physics, repetition rate of $10 \mathrm{~Hz}$ ). The excitation beam was focused on the sample by a cylindrical lens, thus providing a rectangular excitation stripe of width $\cong 1 \mathrm{~mm}$. The DFB emission at room temperature was dispersed by a monochromator and detected by a $\mathrm{Si}$ charge coupled device, providing a spectral resolution of about $0.1 \mathrm{~nm}$.

\section{Results and discussion}

The physical mechanisms of the nanoimprinting process are well-established: upon increasing temperature, a thermoplastic compound is able to reach a rubber-elastic region, still preserving its network of entanglements at a molecular length-scale. This leads to reversible morphological deformations [25]. Then, the material can be driven to its terminal flow region, in which the pressureinduced deformations become irreversible. This region is characterized by a linear dependence of the shear compliance, $J$, on the flow time, $t$, and is mainly due to the viscous molecular flow slipping out of the chain entanglements [25]. Compounds of lower molar-mass can reach the terminal flow region more easily. In particular, we applied this procedure to oligothiophene- $S, S$-dioxides, which exhibit excellent luminescence [14-16] and optical gain [26] performances, obtaining irreversible deformations 


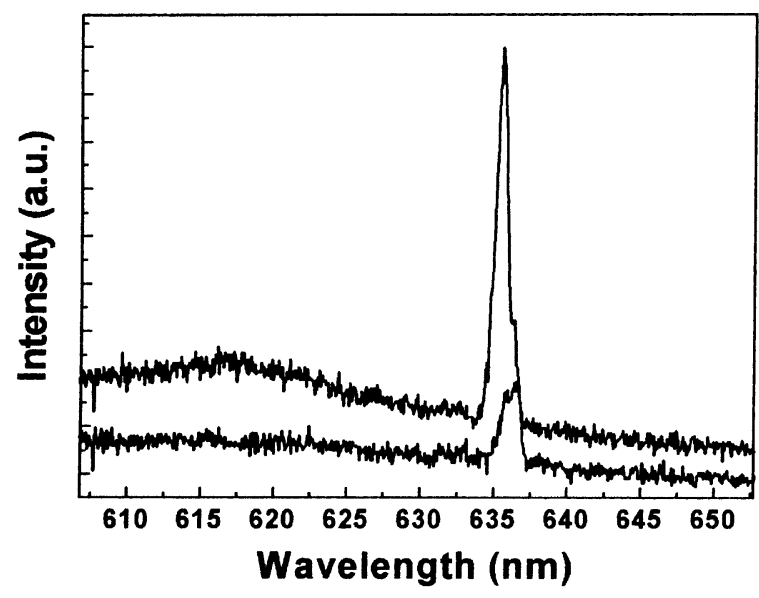

Fig. 4 Emission from the T5oCx-based DFB. the excitation density is 41 and $180 \mu \mathrm{J} / \mathrm{cm}^{2}$ for the bottom and the top spectrum, respectively.

(namely pattern transfer) under force even at room temperature, on timescales ranging between minutes and a few hours [27]. Indeed, these imprinting times, which are larger than those typical of embossing procedures performed at high temperature, provides the time to the organic compound to move under the applied stress and to conform to the mold. We found, for a large number of imprinted oligomers, that the applied force during printing is the key parameter for obtaining a pattern transfer of high quality, and especially for avoiding the detachment of the organic film from the substrate [27]. Similarly to conventional NIL, the imprinting stops as the oligomer has reached a larger contact with the features of the stamp, thus decreasing the effective pressure which acts on the film. This can cause some loss in the aspect-ratio of the imprinted features with respect to that of the master. In this case, the imprinting often transfers a pattern which exhibits two crests corresponding to the two sides of the recessed features of the mold, like in the second step of Fig. 2. Differently, a complete transfer would result in an organic grating perfectly conformed to the master, similarly to the third step of Fig. 2. This can be more easily obtained by larger imprinting times, allowing to easily reach imprinted aspect-ratios of 1 [27].

The nanoimprinted features are quite stable at room temperature. We stored the DFB samples for months without appreciating significant structural relaxation of the realized gratings. This indicates that, upon inducing the deformation of the film by the applied pressure, the material is not sufficiently ductile to restructuring over time, thus remaining in stably imprinted patterns. On the contrary, significant damages can be induced by operation under high excitation densities, mainly by virtue of the intense heating induced by our pumping conditions.

We checked that the PL efficiency, $\eta_{P L}$, of $\mathrm{T} 5 \mathrm{oCx}$ films remained unchanged before and after the patterning process around a value of $18 \%$ [28], thus assessing that no degradation of the luminescence performances of the chromophore occurs upon room temperature nanoimprinting. The patterned $\mathrm{T} 50 \mathrm{Cx}$ films could then be employed as corrugated interfaces for printed, optically pumped DFB devices. The distributed feedback inside a DFB resonator [29] is due to the spatial modulation of the refractive index, $n$, or of the gain of the propagation medium, resulting in a DFB emission which can be doublemode or single-mode, respectively. The lasing wavelength, $\lambda_{m}$, is related to the cavity geometry by the relation:

$$
m \lambda_{m}=2 n_{\text {eff }} \Lambda \text {, }
$$

where $m$ indicates the diffraction order onto the DFB grating, and $n_{\text {eff }}$ is the effective refractive index of the propagating mode. Organic-based DFBs exploited conjugated polymers, [30-32] and blends with commercial dyes [11, 33-37] as active medium, usually deposited on patterned, optically inert substrates (silica or transparent plastics) [32-35]. Instead, we directly control the air/organic interface. A similar approach (solvent-assisted molding), has been recently proposed for conjugated polymers $[38,39]$. The viscosity is decreased by solvents, thus allowing polymers to conform to the mold. Also this method is favoured by the plasticity of the employed compounds, hence it is less applicable to our oligomers.

In previous studies, we demonstrated lasing from printed oligothiophene devices, at the third [40] and second [41] diffraction order ( $\Lambda=600$ and $400 \mathrm{~nm}$, respectively), and with lasing thresholds of 140 and $37 \mu \mathrm{J} \mathrm{cm}$, respectively. Here, we pushed the room-temperature printing to higher resolution, by transferring $100 \mathrm{~nm}$ features $(\Lambda=200 \mathrm{~nm})$, thus realising first-order DFBs. The lasing emission from our DFBs is peaked at $\lambda_{m}=636 \mathrm{~nm}$, with a FWHM of $1.1 \mathrm{~nm}$ (at excitation density larger than $27 \mu \mathrm{J} \mathrm{cm}^{-2}$ ) (Fig. 4). According to Eq. (1), this is provided by the periodic imprinted nanostructure, having an effective refractive index of 1.59. The observed emission is singlemode, which would suggest that the feedback mechanisms is dominated by the periodic variation of the optical gain along the slab, although the spectral shape of the PL peak could resemble a bad-resolved double peak signal. We note that the DFB emission is detected at excitation densities which are lower than the pump-density $\left(0.8 \mathrm{~mJ} \mathrm{~cm}^{-2}\right)$ needed for amplified spontaneous emission from a $\mathrm{T} 5 \mathrm{oCx}$ planar slab under ns-excitation by about a factor of 30 , indicating the effectiveness of the distributed feedback mechanisms. Indeed, this also results in very low pumping thresholds, which are lower than those measured for third and second order devices. On the other side, a slight spontaneous emission background is still appreciable in the DFB emission (especially at higher excitation density, see for instance the top spectrum in Fig. 4), which can be due to a not perfectly-matched resonating condition by lithography-induced distortions, damaging of the nanostructure for the pumping heating, etc.

We could appreciate the dramatic line-narrowing occurring for excitation densities around $30 \mu \mathrm{J} \mathrm{cm}^{-2}$, where the emission spectral width (FWHM) decreases from 97 to about $1 \mathrm{~nm}$ (the minimum line-width that we observed for ns-pumped amplified spontaneous emission from $\mathrm{T} 5 \mathrm{oCx}$ is $9 \mathrm{~nm}$ ). Though the spectra collected above the threshold for line-narrowing allow one to find a roughly linear dependence of the emission intensity on the pump density, unfortunately, due to the instability of the imprinted patterns under high excitation densities, we could not 


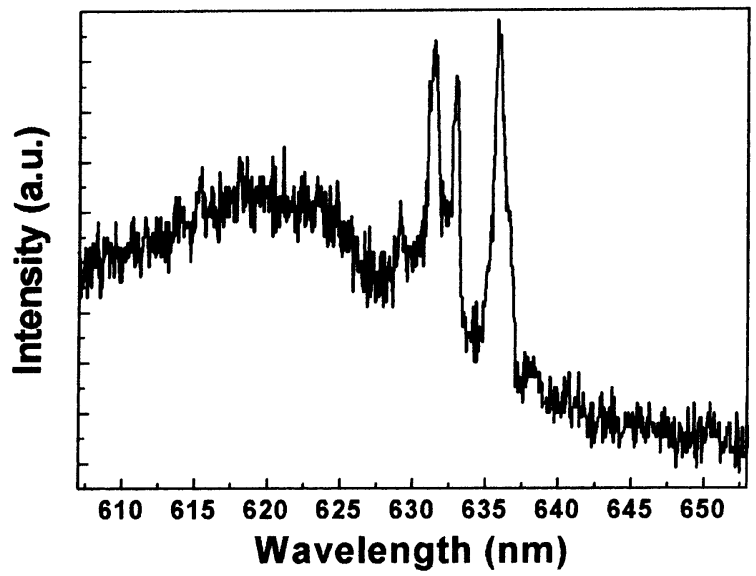

Fig. 5 Multi-mode emission from some regions of the $\mathrm{T} 5 \mathrm{oCx}$ imprinted area (excitation density of $82 \mu \mathrm{J} / \mathrm{cm}^{2}$ ).

determine the complete input-output characteristics of our first-order devices. At higher orders (lower printing resolutions) we realised features on $\mathrm{T} 50 \mathrm{Cx}$ which exhibit higher stability under intense pumping. This difference is likely related to the structural relaxation of the oligomer grating induced by the heating, causing a complete disappearance of $100 \mathrm{~nm}$-features in a few seconds.

Finally, in order to evaluate the homogeneity of the obtained pattern, we collected the DFB emission from different regions of the imprinted sample. In some regions, we found a multi-mode emission, also corresponding to a relatively stronger background PL signal (Fig. 5), clearly indicating a weaker feedback. These effects have been observed also in other DFB devices manufactured by soft lithography [36]. Further work is currently in progress in our laboratory, aiming at further improving the uniformity of room-temperature nanoimprinting on large-areas, and the emission performances of the DFB devices.

\section{Conclusion}

In this work, we reported on first-order oligomer-based DFB devices realised by room-temperature nanoimprint lithography. The DFBs exhibit PL emission with a linewidth of $1.1 \mathrm{~nm}$ at about $636 \mathrm{~nm}$ for excitation fluences larger than $27 \mu \mathrm{J} / \mathrm{cm}^{2}$. Although some issues of our patterning method, such as the amount of process-induced distortions, need a further investigation, these results clearly indicate that substituted oligothiophene- $S, S$-dioxides are promising candidates for solid-state lasers realizable by emerging nanopatterning technologies.

\section{References}

[1] D. Moses, Appl. Phys. Lett. 60 (1992) 3215.

[2] N. Tessler, G. J. Denton, R. H. Friend, Nature 382 (1996) 695.

[3] W. Graupner, G. Leising, G. Lanzani, M. Nisoli, S. De Silvestri, U. Scherf, Phys. Rev. Lett. 76 (1996) 847.

[4] S. V. Frolov, W. Gellerman, M. Ozaki, K. Yoshino, Z. V. Vardeny, Phys. Rev. Lett. 78 (1997) 729.

[5] G. J. Denton, N. Tessler, M. A. Stevens, R. H. Friend, Adv. Mater. 9 (1997) 547
[6] S. Stagira, M. Nisoli, G. Cerullo, M. Zavelani-Rossi, S. De Silvestri, G. Lanzani, W. Graupner, G. Leising, Chem. Phys. Lett. 289 (1998) 205. [7] B.Schweitzer, G. Wengmann, H. Giessen, D. Hertel, H. Bassler, R. F. Mahrt, Appl. Phys. Lett. 72 (1998) 2933.

[8] S. V. Frolov, M. Shkunov, Z. V. Vardeny, K. Yoshino, Phys. Rev. B 56 (1997) 4363.

[9] V. G. Kozlov, V. Bulović, P. E. Burrows, S. R. Forrest, Nature 389 (1997) 362.

[10] M. Bergreen, A. Dodabalapur, R. E. Slusher, Z. Bao, Nature 389 (1997) 466.

[11] M. Bergreen, A. Dodabalapur, R. E. Slusher, Appl. Phys. Lett. 71 (1997) 2230.

[12] S. Stagira, M. Zavelani-Rossi, M. Nisoli, S. De Silvestri, G. Lanzani, C. Zenz, P. Mataloni, G. Leising, Appl. Phys. Lett. 73 (1998) 2860.

[13] M. D. McGehee, M. A. Diaz-García, F. Hide, R. Gupta, E. K. Miller, D. Moses, A. J. Heeger, Appl. Phys. Lett. 72 (1998) 1536.

[14] M. Anni, G. Gigli, V. Paladini, R. Cingolani, G. Barbarella, L. Favaretto, G. Sotgiu, M. Zambianchi, Appl. Phys. Lett. 77 (2000) 2458.

[15] G. Gigli, O. Inganas, M. Anni, M. De Vittorio, R. Cingolani, G. Barbarella, L. Favaretto, Appl. Phys. Lett. 78 (2001) 1493.

[16] G. Barbarella, L. Favaretto, M. Zambianchi, O. Pudova, C. Arbizzani, A. Bongini, M. Mastragostino, Adv. Mater. 10 (1998) 551.

[17] S. Y. Chou, P. R. Krauss, P. J. Renstrom, Appl. Phys. Lett. 67 (1995) 3114; Science 272 (1996) 85.

[18] Y. Xia, G. M. Whitesides, Angew. Chem. Int. Ed. 37 (1998) 550

[19] M. Li, L. Chen, S. Y. Chou, Appl. Phys. Lett. 78 (2001) 3322.

[20] L. Guo, P. R. Krauss, S. Y. Chou, Appl. Phys. Lett. 71 (1997) 1881.

[21] Z. Yu, S. J. Schablitsky, S. Y. Chou, Appl. Phys. Lett. 74 (1999) 2381 .

[22] S. Y. Chou, P. R. Krauss, W. Zhang, L. Guo, L. Zhuang, J. Vac. Sci. Technol. 15 (1997) 2897.

[23] J. Wang, X. Sun, L. Chen, S. Y. Chou, Appl. Phys. Lett. 75 (1999) 2767.

[24] M. Behl, J. Seekamp, S. Zankovych, C. M. Sotomayor Torres, R. Zentel, J. Ahopelto, Adv. Mater. 14 (2002) 588.

[25] G. Strobl, The Physics of Polymers, Springer, Berlin 1997.

[26] D. Pisignano, M. Anni, G. Gigli, R. Cingolani, M. Zavelani-Rossi, G. Lanzani, G. Barbarella, L. Favaretto, Appl. Phys. Lett. 81 (2002) 3534.

[27] D. Pisignano, L. Persano, M. F. Raganato, P. Visconti, R. Cingolani, G. Barbarella, L. Favaretto, G. Gigli, Adv. Mater. 16 (2004) 525.

[28] For the measurement of the absolute quantum efficiency, we used as exciting source a He-Cd laser ( $\lambda=325 \mathrm{~nm})$, and we collected the PL signal in an integrating sphere, by a fibre-coupled charge coupled device (Ocean Optics, FL). The number of PL photons per absorbed photon emitted by the untextured and the patterned films was determined by accounting for those photons which are not absorbed by the sample at their first incidence, and are absorbed after successive reflections on the surface of the integrating sphere. Complete details on this measurement can be found elsewhere: D. Pisignano, A. Melcarne, D. Mangiullo, R. Cingolani, G. Gigli, J. Vac. Sci. Technol. B 22 (2004) 185.

[29] H. Kolgelnik, V. Shank, Appl. Phys. Lett. 18 (1971) 152.

[30] N. Moll, R. F. Mahrt, C. Bauer, H. Giessen, B. Schnabel, E. B. Kley, U. Scherf, Appl. Phys. Lett. 80 (2002) 734.

[31] S. Riechel, C. Kallinger, U. Lemmer, J. Feldmann, A. Gombert, V. Wittwer, U. Scherf, Appl. Phys. Lett. 77 (2000) 2310.

[32] C. Kallinger, M. Hilmer, A. Haugeneder, M. Perner, W. Spirkl, U. Lemmer, J. Feldmann, U. Scherf, K. Müllen, A. Gombert, V. Wittwer, Adv. Mater. 10 (1998) 920.

[33] M. Bergreen, A. Dodabalapur, R. Slusher, A. Timko, O. Nalamasu Appl. Phys. Lett. 72 (1998) 410.

[34] J. A. Rogers, M. Meier, A. Dodabalapur, Appl. Phys. Lett. 73 (1998) 1766.

[35] J. A. Rogers, M. Meier, A. Dodabalapur, E. J. Laskowski, M. A. Cappuzzo, Appl. Phys. Lett. 74 (1999) 3257.

[36] B. J. Scott, G. Wirnsberger, M. D. McGehee, B. F. Chmelka, G. D. Stucky, Adv. Mater. 13 (2001) 1231

[37] S. Riechel, U. Lemmer, J. Feldmann, S. Berleb, A. G. Mückl, W. Brütting, A. Gombert, V. Wittwer, Opt. Lett. 26 (2001) 593.

[38] M. Gaal, C. Gadermaier, H. Plank, E. Moderegger, A. Pogantsch, G. Leising, E. J. W. List, Adv. Mater. 15 (2003), 1165.

[39] J. R. Lawrence, G. A. Turnbull, I. D. W. Samuel, Appl. Phys. Lett. 82 (2003) 4023.

[40] D. Pisignano, L. Persano, P. Visconti, R. Cingolani, G. Gigli, G. Barbarella, L. Favaretto, Appl. Phys. Lett. 83 (2003) 2545.

[41] D. Pisignano, L. Persano, E. Mele, P. Visconti, R. Cingolani, G. Gigli, G. Barbarella, L. Favaretto, Opt. Lett., in press. 